\title{
Author Correction: Temporal Variation of White Rhino Dung Odours
}

\section{Courtney Marneweck ${ }^{1}$ (D) - Andreas Jürgens ${ }^{2} \cdot$ Adrian M. Shrader $^{1,3}$}

Published online: 8 March 2019

(C) Springer Science+Business Media, LLC, part of Springer Nature 2019

\section{Author Correction: Journal of Chemical Ecology (2017) 43:955-965 https://doi.org/10.1007/s10886-017-0890-4}

The original version of this article unfortunately contained some mistakes. Firstly, Fig. 5 listed a contaminant. The correct version of Fig. 5 can be found here. Second, an incorrect version of the Supplementary Table A1 was mistakenly included. A corrected and updated version of Supplementary Table A1 can be found here as well, pertaining specifically to the important compounds highlighted in Fig. 5 and with additional chemical information included.

The authors apologize for this oversight and for any confusion it may have caused.

The online version of the original article can be found at https://doi.org/ 10.1007/s10886-017-0890-4

Courtney Marneweck

courtney.marneweck@gmail.com

1 School of Life Sciences, University of KwaZulu-Natal, Durban, South Africa

2 Chemical Plant Ecology, Technische Universität Darmstadt, Darmstadt, Germany

3 Present address: Mammal Research Institute, Department of Zoology \& Entomology, University of Pretoria, Pretoria, South Africa 
a) Territorial wet

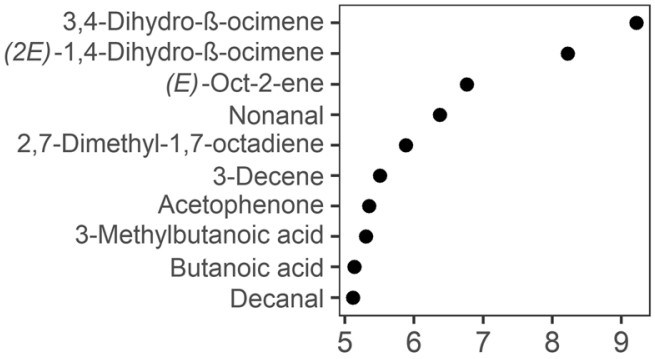

c) Non-territorial wet

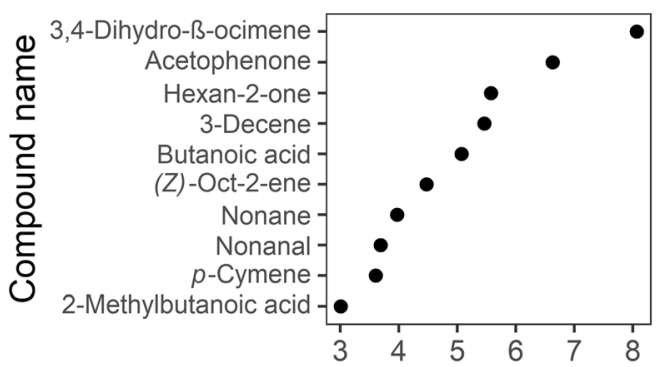

e) Oestrous wet

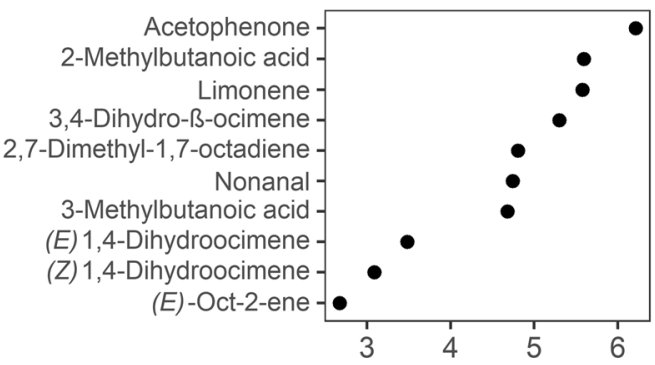

g) Non-oestrous wet

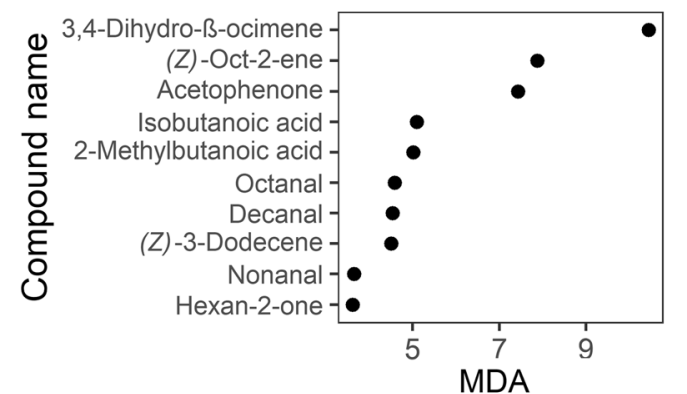

Fig. 5 The importance of volatile organic compounds (VOCs) for distinguishing the age of dung from territorial males, a) wet season, b) dry season; non-territorial males c) wet season, d) dry season; estrus b) Territorial dry

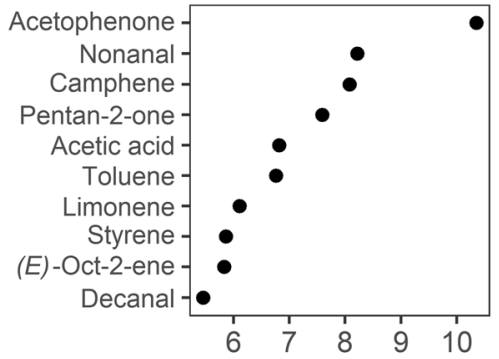

d) Non-territorial dry

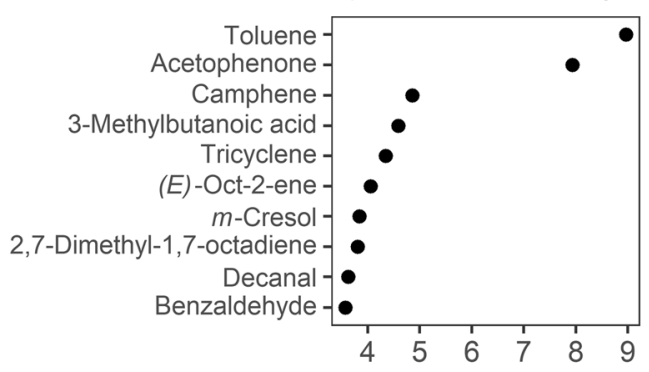

f) Oestrous dry

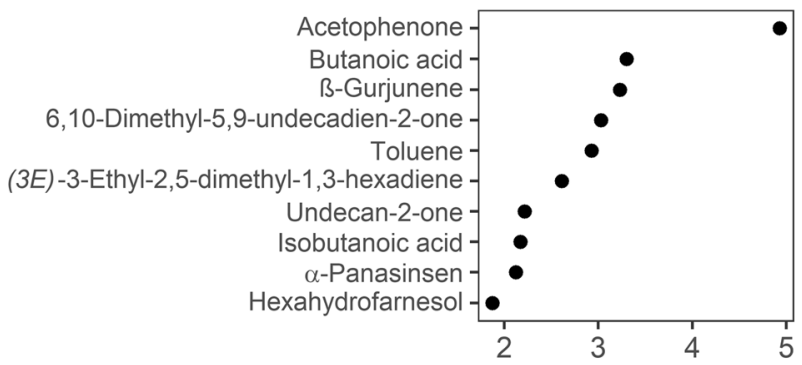

h) Non-oestrous dry

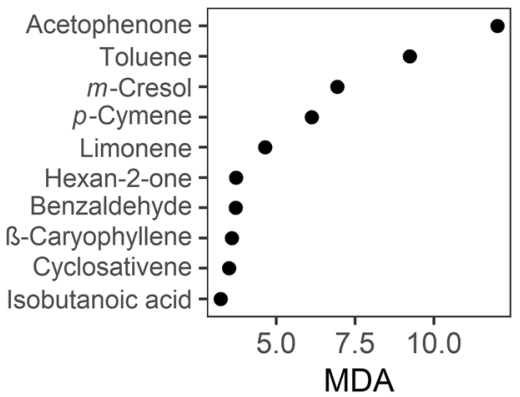

females e) wet season, f) dry season; and non-estrus females $\mathbf{g}$ ) wet season, h) dry season. Importance was based on mean decrease in accuracy (MDA). Only the top ten compounds are presented in the figure 
Table A1 Chemical information pertaining to the tentative identification of compounds listed in Fig. 5 (the importance of volatile organic compounds for distinguishing the age of dung)

\begin{tabular}{|c|c|c|c|c|c|c|}
\hline Compound & $\begin{array}{l}\text { Retention } \\
\text { time } \\
(\mathrm{min} .)\end{array}$ & $\begin{array}{l}\text { Retention } \\
\text { index RI } \\
\text { (calc.) }\end{array}$ & $\begin{array}{l}\text { Retention } \\
\text { Index RI } \\
\text { (lit.) }\end{array}$ & $\begin{array}{l}\text { Observed Match and R. } \\
\text { Match values }\end{array}$ & $\begin{array}{l}\text { Lit. Source of RI / Lit. Source } \\
\text { of MS (NIST 2013) }\end{array}$ & $\begin{array}{l}\text { MS-Spectrum in } \\
\text { Sample (most } \\
\text { abundant peaks) }\end{array}$ \\
\hline
\end{tabular}

\section{Alkanes}

Nonane ${ }^{c}$

Alkenes

$\begin{array}{llll}\text { (E)-2-Octene }^{\mathrm{b}} & 4.32 & 799 & 798 \\ \text { (Z)-2-Octene }^{\mathrm{b}} & 4.38 & 802 & 817 \\ \text { (E)-3-Decene }^{\mathrm{a}} & 7.77 & 961 & -\end{array}$

(Z)-3-Dodecene ${ }^{\text {a }}$

13.06

1193

Aliphatic Aldehydes

\begin{tabular}{|c|c|c|c|}
\hline Octanal $^{c}$ & 8.45 & 991 & 1004 \\
\hline Nonanal $^{\mathrm{c}}$ & 10.98 & 1112 & 1103 \\
\hline Decanal $^{\mathrm{c}}$ & 13.17 & 1198 & 1205 \\
\hline \multicolumn{4}{|l|}{ Aliphatic Ketones } \\
\hline Pentan-2-one ${ }^{b}$ & 2.43 & 664 & 691 \\
\hline Hexan-2-one ${ }^{b}$ & 4.03 & 780 & 790 \\
\hline Undecan-2-one $^{b}$ & 14.61 & 1269 & 1291 \\
\hline \multicolumn{4}{|l|}{ Aliphatic Acids } \\
\hline Acetic acid $^{\mathrm{c}}$ & 1.82 & 601 & 600 \\
\hline Isobutanoic acid ${ }^{\mathrm{b}}$ & 3.39 & 740 & 765 \\
\hline Butanoic acid $^{\mathrm{b}}$ & 4.28 & 797 & 790 \\
\hline 3-Methylbutanoic acid ${ }^{\mathrm{b}}$ & 4.98 & 831 & 831 \\
\hline 2-Methylbutanoic acid ${ }^{\mathrm{b}}$ & 5.40 & 851 & 853 \\
\hline \multicolumn{4}{|l|}{ Aromatic compounds } \\
\hline Toluene $^{\mathrm{c}}$ & 3.73 & 761 & 762 \\
\hline$p$-Xylene ${ }^{\mathrm{c}}$ & 5.70 & 866 & 869 \\
\hline Styrene $^{\mathrm{c}}$ & 6.22 & 891 & 894 \\
\hline Benzaldehyde $^{c}$ & 7.77 & 960 & 948 \\
\hline$p$-Cymene ${ }^{\mathrm{b}}$ & 9.26 & 1030 & 1060 \\
\hline Acetophenone $^{\mathrm{c}}$ & 10.14 & 1073 & 1062 \\
\hline$m$-Cresol ${ }^{\mathrm{c}}$ & 10.53 & 1093 & 1073 \\
\hline \multicolumn{4}{|l|}{ Terpenoids } \\
\hline Tricyclene $^{\mathrm{b}}$ & 6.98 & 925 & 923 \\
\hline Camphene $^{\mathrm{b}}$ & 7.62 & 954 & 945 \\
\hline 3,4-Dihydro- $\beta$-ocimene ${ }^{b}$ & 7.40 & 944 & 939 \\
\hline (E)-1,4-Dihydro- $\beta$-ocimene ${ }^{\mathrm{b}}$ & 8.33 & 985 & 990 \\
\hline (Z)-1,4-Dihydro- $\beta$-ocimene ${ }^{\mathrm{b}}$ & 8.64 & 999 & 990 \\
\hline
\end{tabular}

Match: 813 R.Match: 890

Match: 799 R.Match: 886 Xu et al. (2003)

Match: 779 R.Match: 853 Garcia-Estaban et al. (2004)

Match: 801 R.Match: $854 \quad 41=999$ | 55=868 | 69=691 | 69=999| 41=732| $56=630|43=483| 70=482 \quad 70=651|55=615|$ | $39=320 \mid 42=311$ | $56=348|43=226|$ $29=273|27=263| \quad 140=222 \mid 39=$ $207|57=199| 67=$ 194

Match: 828 R.Match: $88655=999|41=949| 56=898|\quad 55=999| 43=751 \mid$ $69=881|43=715| 70=650 \quad 41=731|69=725|$ $|57=582| 83=396 \mid$ $42=331 \mid 29=287$ |

$56=667|70=551|$

$83=410 \mid 105=$ $409|77=407| 84=$ 363 |
Match: 786 R.Match: 903 Moio et al. (1996)

Match: 808 R.Match: 850 Flamini et al. (2007)

Match: 862 R.Match: 887 Baccouri et al. (2007)

Match: 848 R.Match: 859 Macku and Shibamoto (1991)

Match: 856 R.Match: 905 Wu et al. (2007)

Match: 652 R.Match: 858 Adams (1999)

Match: 901 R.Match: 920 Whetstine et al. (2005)

Match: 808 R.Match: 870 Mateo and Zumalacarregui (1996)

Match: 813 R.Match: 820 Quijano et al. (2007)

Match: 664 R.Match: 724 Ventanas et al. (2008)

Match: 690 R.Match: 745 Kondjoyan et al. (1997)

Match: 923 R.Match: 950 Moon et al. (2006)

Match: 824 R.Match: 922 Helmig et al. (1996)

Match: 773 R.Match: 837 Insausti et al. (2005)

Match: 817 R.Match: 892 Jordan et al. (2001)

Match 643 R.Match: 683 Marongiu et al. (2004)

Match: 914 R.Match: 946 Schwambach and Peterson (2006)

Match: 925 R.Match: 934 Re-Poppi and Santiago-Silva (2002)

Match: 876 R.Match: 910 Pino et al. (2005)

Match: 919 R.Match: 962 Zhang et al. (2005)

Match: 915 R.Match: 916 Diaz and Kite (2002)

Match: 899 R.Match: 959 Buchin et al. (2002)

Match: 866 R.Match: 928 Buchin et al. (2002) 
Table A1 (continued)

\begin{tabular}{|c|c|c|c|c|c|c|}
\hline Compound & $\begin{array}{l}\text { Retention } \\
\text { time } \\
\text { (min.) }\end{array}$ & $\begin{array}{l}\text { Retention } \\
\text { index RI } \\
\text { (calc.) }\end{array}$ & $\begin{array}{l}\text { Retention } \\
\text { Index RI } \\
\text { (lit.) }\end{array}$ & $\begin{array}{l}\text { Observed Match and } \mathrm{R} \text {. } \\
\text { Match values }\end{array}$ & $\begin{array}{l}\text { Lit. Source of RI/Lit. Source } \\
\text { of MS (NIST 2013) }\end{array}$ & $\begin{array}{l}\text { MS-Spectrum in } \\
\text { Sample (most } \\
\text { abundant peaks) }\end{array}$ \\
\hline Unknown terpenoid $\mathrm{A}^{\mathrm{a}}$ & 7.07 & 929 & - & - & - & $\begin{array}{c}55=999|41=981| \\
67=847|39=653| \\
68=473|95=406| \\
53=272|56=262| \\
69=260|81=245|\end{array}$ \\
\hline Limonene $^{\mathrm{c}}$ & 9.41 & 1037 & 1039 & Match: 907 R.Match: 927 & Blagojevic et al. (2006) & \\
\hline$\beta$-Caryophyllene ${ }^{c}$ & 17.72 & 1442 & 1440 & Match: 904 R.Match: 908 & Bylaite and Meyer (2006) & \\
\hline Cyclosativene $^{\mathrm{b}}$ & 16.73 & 1393 & 1370 & Match: 886 R.Match: 893 & Andrade et al. (2007) & \\
\hline$\beta$-Gurjunene ${ }^{b}$ & 17.91 & 1451 & 1428 & Match: 911 R.Match: 936 & Saroglou et al. (2007) & \\
\hline Geranylacetone $^{\mathrm{b}}$ & 17.96 & 1453 & 1445 & Match: 756 R.Match: 819 & Dhanda et al. (2003) & \\
\hline Unknown terpenoid $\mathrm{B}^{\mathrm{a}}$ & 19.49 & 1533 & - & - & - & $\begin{array}{c}161=999 \mid 122=670 \\
|107=588| 105= \\
399|91=389| 81 \\
=355|204=338| \\
79=305 \mid 41=299 \\
|93=273|\end{array}$ \\
\hline Unknown terpenoid $\mathrm{C}^{\mathrm{a}}$ & 23.17 & 1779 & - & - & - & $\begin{array}{c}32=999|43=844| \\
70=736 \mid 55=669 \\
|69=621| 71= \\
567|57=564| 41 \\
=562|56=312| \\
58=289 \mid\end{array}$ \\
\hline
\end{tabular}

a comparison of MS with published data

${ }^{\mathrm{b}}$ comparison of MS and retention time with published data

${ }^{c}$ comparison of MS and retention time with authentic standard

\section{References}

Adams RP (1999) Systematics of multi-seeded eastern hemisphere Juniperus based on leaf essential oils and RAPD DNA fingerprinting. Biochem Syst Ecol 27:709-725

Andrade EHA, Oliveira J, Zoghbi MGB (2007) Volatiles of Anaxagorea dolichocarpa Spreng. \& Sandw. and Annona densicoma Mart. Growing Wild in the State of Para, Brazil. Flavour Fragr J 22:158160

Baccouri B, Ben Temime S, Campeol E, Cioni PL, Daoud D, Zarrouk M (2007) Application of solid-phase microextraction to the analysis of volatile compounds in virgin olive oils from five new cultivars. Food Chem 102:850-856

Blagojevic P, Radulovic N, Palic R, Stojanovic G (2006) Chemical composition of the essential oils of Serbian wild-growing Srtemisia absinthium and Artemisia vulgaris. J Agric Food Chem 54:4780-4789

Buchin S, Salmon J-C, Carnat A-P, Berger T, Bugaud C, Bosset JO (2002) Identification de composes monoterpeniques, sesquiterpeniques et benzeniques dans un lait d'alpage tres riche en ces substances, Mitt. Lebensmittelunters. Hyg. 93:199-216

Bylaite E, Meyer AS (2006) Characterisation of volatile aroma compounds of orange juices by three dynamic and static headspace gas chromatography techniques. Eur Food Res Technol 222:176-184

Dhanda JS, Pegg RB, Shand PJ (2003) Saskatchewan specialty livestock value-added program. Saskatchewan agri-food innovation fund (AFIF) Project \#98000016
Diaz A, Kite GC (2002) A comparison of the pollination ecology of Arum maculatum and A. italicum in England, Watsonia. 24:171-181

Flamini G, Cioni PL, Morelli I, Bader A (2007) Essential oils of the aerial parts of three Salvia species from Jordan: Salvia lanigera, S. spinosa and S. syriaca. Food Chem, 100:732-735

Garcia-Estaban M, Ansorena D, Astiasaran I, Ruiz J (2004) Study of the effect of different fiber coatings and extraction conditions on dry cured ham volatile compounds extracted by solid-phase microextraction (SPME). Talanta 64:458-466

Helmig D, Pollock W, Greenberg J, Zimmerman P (1996) Gas chromatography mass spectrometry analysis of volatile organic trace gases at Mauna Loa Observatory, Hawaii. J Geophys Res 101:1469714710

Insausti K, Goni V, Petri E, Gorraiz C, Beriain MJ (2005) Effect of weight at slaughter on the volatile compounds of cooked beef from Spanish cattle breeds. Meat Sci 70:83-90

Jordan MJ, Shaw PE, Goodner KL (2001) Volatile components in aqueous essence and fresh fruit of Cucumis melo cv. Athena (muskmelon) by GC-MS and GC-O. J Agric Food Chem 49:59295933

Kondjoyan N, Viallon C, Berdague JL, Daridan D, Simon M-N, Legault C (1997) Analyse comparative de la fraction volatile de jambons secs de porcs Gascon et Large-White x Landrace Francais. J Rech CNRS 29:405-410

Macku C, Shibamoto T (1991) Headspace volatile compounds formed from heated corn oil and corn oil with glycine. J Agric Food Chem 39:1265-1269 
Marongiu B, Piras A, Porcedda S (2004) Comparative analysis of the oil and supercritical $\mathrm{CO} 2$ extract of Elettaria cardamomum (L.) maton. J Agric Food Chem 52:6278-6282

Mateo J, Zumalacarregui JM (1996) Volatile compounds in chorizo and their changes during ripening. Meat Sci 44:255-273

Moio L, Rillo L, Ledda A, Addeo F (1996) Odorous constituents of ovine milk in relationship to diet. J Dairy Sci 79:1322-1331

Moon S-Y, Cliff MA, Li-Chan ECY (2006) Odour-active components of simulated beef flavour analysed by solid phase microextraction and gas chromatography-mass spectrometry and -olfactometry. Food Res Int 39:294-308

Pino JA, Mesa J, Munoz Y, Marti MP, Marbot R (2005) Volatile components from mango (Mangifera indica L.) cultivars. J Agric Food Chem 53:2213-2223

Quijano CE, Salamanca G, Pino JA (2007) Aroma volatile constituents of Colombian varieties of mango (Mangifera indica L.). Flavour Fragr J 22:401-406

Re-Poppi N, Santiago-Silva MR (2002) Identification of polycyclic aromatic hydrocarbons and methoxylated phenols in wood smoke emitted during production of charcoal. Chromatographia $55: 475-481$
Saroglou V, Marin PD, Rancic A, Veljic M, Skaltsa H (2007) Composition and antimicrobial activity of the essential oil of six Hypericum species from Serbia. Biochem Syst Ecol 35:146-152

Schwambach SL, Peterson DG (2006) Reduction of Stale Flavor Development in Low-Heat Skim Milk Powder via Epicatechin Addition. J Agric Food Chem 54:502-508

Ventanas S, Estevez M, Andres AI, Ruiz J (2008) Analysis of volatile compounds of Iberian dry-cured loins with different intramuscular fat contents using SPME-DED. Meat Sci 79:172-180

Whetstine MEC, Cadwallader KR, Drake MA (2005) Characterization of aroma compounds responsible for the rosy/floral flavor in cheddar cheese. J Agric Food Chem 53:3126-3132

Wu S, Zorn H, Krings U, Berger RG (2007) Volatiles from submerged and surface-cultured beefsteak fungus, Fistulina hepatica. Flavour Fragr J 22:53-60

$\mathrm{Xu}$ X et al. (2003) Comprehensive two-dimensional gas chromatography $(\mathrm{GC} * \mathrm{GC})$ measurements of volatile organic compounds in the atmosphere. Atmos Chem Phys, 3:665-682

Zhang F, Xu Q, Fu S, Ma X, Xiao H, Liang X (2005) Chemical constituents of the essential oil of Asarum forbesii Maxim (Aristolochiaceae). Flavour Fragr J 20:318-320 